\title{
MALDI-TOF Mass Spectrometric Analysis of Brain Tumor Cyst Fluid Reveals a Protein Peak Corresponding to ApoC1 and LuzP6
}

\author{
Mathias Groll1', Jochen Frenzel2, Matthias Krause'1, Anne Schänzer ${ }^{3}$, Wolf Müller ${ }^{4}$, \\ Klaus Eschrich ${ }^{2}$, Ulf Nestler ${ }^{*}$ \\ ${ }^{1}$ Department of Neurosurgery, University Hospital Leipzig, Leipzig, Germany \\ ${ }^{2}$ Rudolf-Schönheimer-Institute of Biochemistry, Medical Faculty, University of Leipzig, Leipzig, Germany \\ ${ }^{3}$ Institute of Neuropathology, Justus Liebig University Giessen, Giessen, Germany \\ ${ }^{4}$ Institute of Neuropathology, University Hospital Leipzig, Leipzig, Germany \\ Email: *ulf.nestler@medizin.uni-leipzig.de
}

How to cite this paper: Groll, M., Frenzel, J., Krause, M., Schänzer, A., Müller, W., Eschrich, K. and Nestler, U. (2018) MALDITOF Mass Spectrometric Analysis of Brain Tumor Cyst Fluid Reveals a Protein Peak Corresponding to ApoC1 and LuzP6. Open Journal of Modern Neurosurgery, 8, 251-263. https://doi.org/10.4236/ojmn.2018.83021

Received: April 12, 2018

Accepted: June 23, 2018

Published: June 26, 2018

Copyright $(9) 2018$ by authors and Scientific Research Publishing Inc. This work is licensed under the Creative Commons Attribution International License (CC BY 4.0).

http://creativecommons.org/licenses/by/4.0/

\begin{abstract}
Objectives: SELDI-TOF and MALDI-TOF mass spectrometry (MS) are laser desorption technologies that allow for proteomic examination of molecular masses in small amounts of samples. In a precedent study, the feasibility of SELDI-TOF MS assessment of proteins in cerebrospinal fluid and tumor cyst fluid had been shown. In the present study, we analyzed whether MALDI-TOF MS examination of these fluids leads to comparable results. Methods: During neurosurgical intervention, cyst fluids from 24 glioblastomas and 15 metastases were collected. As control, cerebrospinal fluid samples from 23 patients were obtained. The samples were prepared using a protocol optimized for MALDI-TOF MS. Mass spectra were recorded and peaks were extracted, characterized by masses and relative intensities. These peaks were analyzed for statistically significant differences between the diagnosis groups and compared to SELDI-TOF MS data. Results: 41 protein peaks known from the SELDI-TOF MS analysis could be confirmed by MALDI-TOF MS, and the cellular expression of the proteins LuzP6 and ApoC1, corresponding to the protein peaks 6433 and 6632, was shown immunohistochemically in glioblastoma tissue. The MALDI-TOF spectrometry extends the range of analysis down to $1.4 \mathrm{kDa}$, whereas the upper detection limit lies below $23 \mathrm{kDa}$. Discussion: The presented proteomic approach yields an inventory of protein masses, found in the tumor cyst at the time of puncture. It does not reveal pathophysiologic, metabolic or secretory pathways that lead to the presence of proteins in the cyst. These have to be assessed immunohistochemically or on mRNA level in the surrounding tumor cells. Conclusion: MALDI-TOF MS of
\end{abstract}


tumor cyst fluid discloses protein sizes, overexpressed or lost in tumor tissue. A thorough proteomic work-up is needed to identify the underlying proteins and metabolic pathways.

\section{Keywords}

Cerebrospinal Fluid, Cerebral Metastasis, Glioblastoma, Mass Spectrometry, Tumor Cyst

\section{Introduction}

Tumor cysts occur in a multitude of brain tumors. While in entities such as hemangioblastoma or oligodendroglioma cysts can be considered to be pathognomonic, the most frequent occurrence is in glioblastoma, metastasis or meningioma [1]. For glioblastoma tissue, significant differences of protein content between tumor cyst fluid and control cerebrospinal fluid (CSF) have been shown [2]. Since the cyst often forms a closed compartment, surrounded by tumor cells, proteomic analysis of the cyst fluid may help in describing pathophysiologic pathways, leading to cyst formation or tumor growth.

Tumor cyst fluid is only available in small amounts with very low concentration of proteins. The surface enhanced laser desorption ionisation mass spectrometry (SELDI-TOF MS), a highly sophisticated variant of matrix assisted laser desorption ionisation (MALDI-TOF MS), allows to assess directly cyst fluid protein content in as few as 2 microliters of specimen [2]. However, also standard MALDI-TOF mass spectrometry should be applicable to cyst fluid, if samples are desalted and concentrated properly before mass spectrometric analysis.

In the present study, assessment of brain tumor cysts by MALDI-TOF MS was compared to the published SELDI-TOF MS results. The subsequent identification of potential proteins corresponding to the detected mass peaks was performed by an internet database search using the Expasy tagident tool. The $\mathrm{m} / \mathrm{z}$ ratio of the peak was entered, allowing for a deviation of $+/-0.3 \%$, according to the accuracy of the SELDI-TOF and MALDI-TOF MS techniques [2].

\section{Materials and Methods}

The ethic committee of the Faculty of Medicine at the University of Leipzig had voted positively to the study prior to collection of specimens and to analysis of patient data. Informed consent was obtained from the patients before neurosurgical intervention. The collection period comprised the three years 2014, 2015 and 2016.

In total, 24 glioblastoma cyst fluids (14 newly diagnosed, 10 recurrent), 15 fluid samples from cerebral tumors originating outside the CNS (5 lung cancer, 3 melanoma, 2 lymphoma, 1 ovarian carcinoma, 1 chorion carcinoma, 1 urothel carcinoma, 1 renal cell carcinoma and 1 adenocarcinoma of unknown origin) and 23 reference CSF samples were examined. Intraoperatively, the cyst was 
punctured under sonographic guidance before starting tumor dissection. The aspirated fluid was cooled down and frozen to $-20^{\circ} \mathrm{C}$. CSF samples from lumbar punction or ventricular drainage were treated similarly. After thawing for the first time, samples were centrifuged at $1000 \times \mathrm{g}$ for 5 minutes at room temperature. The supernatant was distributed into aliquots and re-cryoconservated at $-20^{\circ} \mathrm{C}$ until use. After thawing an aliquot, tumor cyst fluid supernatant was diluted 1:5 in $0.5 \mathrm{M}$ TRIS buffer $\mathrm{pH}$ 6.8, while CSF supernatant was used undiluted. $30 \mu \mathrm{l}$ of these samples were mixed with $60 \mu \mathrm{l}$ binding buffer and $10 \mu \mathrm{l}$ magnetic bead suspension for protein preparation by weak cation exchange (WCX kit, Bruker Daltonics, Bremen). The subsequent washing steps were performed according to the instructions of the manufacturer. Finally, $1 \mu \mathrm{l}$ of the cleaned specimen was spotted on a ground steel target, air dried and covered with $1 \mu \mathrm{l}$ of cinnamic acid matrix ( $4 \mathrm{mg} / \mathrm{ml}$ HCCA in a 1:1 mixture of ACN with $0.1 \%$ TFA). Mass spectra were recorded by MALDI-TOF MS from 1500 to 16,600 mass over charge ratio $(\mathrm{m} / \mathrm{z})$ using an Autoflex II spectrometer (Bruker Daltonics) in linear positive mode. 500 shots were accumulated per spectrum (Figure 1). A mixture of six peptides was utilized for external calibration covering a mass range from 1620.86 to $12,360.97 \mathrm{~m} / z$. For internal calibration, representative samples were mixed with the peptides and measured again. The raw spectra were processed by baseline subtraction and a slight smoothing with Flex analysis 2.4 (Bruker Daltonics). From each spectrum peaks were extracted.



Figure 1. Screen shot showing a MALDI-TOF mass spectrum from glioblastoma cyst fluid. After labeling of peaks by hand, the software calculates the corresponding mass/charge ratio $\mathrm{m} / \mathrm{z}$ (molecular weight in Dalton, for $\mathrm{z}=1$ ) and the intensity. 
The thresholds for peak assignment were a signal to noise ratio of least 6 and a peak width (in half height of maximum) of $0.1 \mathrm{~m} / \mathrm{z}$. Peaks with very low relative intensities were omitted. The different peak intensities in one sample were compared to that of the highest peak and expressed as relative intensities, ranging from 0 to 1 .

\section{Data Analysis}

A list of peaks was generated for all three diagnosis groups (glioblastoma, metastasis, CSF), sorted by weight, comparing the samples of one group. Protein peak masses $(\mathrm{m} / \mathrm{z})$ that differed less than $0.3 \%$ between samples were considered to stem from the same protein. For each of these protein sizes, the percentage of positive samples per diagnosis group and the mean relative intensity in the group were calculated. In this way, 148 protein bands were found in glioblastoma cyst fluid, 125 in metastases and 94 in CSF. The protein peaks were then sorted according to their mean relative intensity from highest to lowest (Table 1). The percentage of samples in which the same protein band was found, was compared between the diagnosis groups by a two sided Fishers exact test of a $2 \times$ 2 contingency table.

For identification of proteins that correspond to the mass peaks, the $\mathrm{m} / \mathrm{z}$ ratio of the peaks was entered into the Expasy tagident tool. The resulting characteristics of potential proteins given by Expasy tagident were then used for an extended PubMed literature search, combining the name of the protein with "brain", "tumor", "glioblastoma”, "metastasis" and "CSF”.

\section{Immunohistochemistry}

For the two protein candidates, potentially corresponding to the spectrometric peak at $\mathrm{m} / \mathrm{z}$ 6433, rabbit polyclonal antibodies were employed. In anti-ApoC1

Table 1. MALDI-TOF MS protein peaks with the highest mean relative intensity and their percentage of positive samples in the three diagnosis groups.

\begin{tabular}{|c|c|c|c|c|c|c|c|c|c|c|c|c|c|}
\hline \multirow{2}{*}{ Nr. } & \multirow{2}{*}{ found in } & \multicolumn{2}{|c|}{ CSF } & \multirow[b]{2}{*}{$\%(n=23)$} & \multicolumn{2}{|c|}{ metastases } & \multicolumn{4}{|c|}{ glioblastoma } & \multicolumn{3}{|c|}{$\mathrm{p}$-value } \\
\hline & & Dalton & rel int & & Dalton & rel int & $\%(n=15)$ & Dalton & rel int & $\%(\mathrm{n}=24)$ & CSF-met & CSF-gbm & met-gbm \\
\hline 1 & CSF & 3513 & 0.86 & 91.3 & 3511 & 0.05 & 13.3 & 3511 & 0.07 & 12.5 & $<0.0001$ & $<0.0001$ & ns \\
\hline 2 & CSF & 3953 & 0.47 & 91.3 & 3955 & 0.04 & 6.7 & - & - & 0 & $<0.0001$ & $<0.0001$ & ns \\
\hline 3 & gbm & 6436 & 0.03 & 21.7 & 6436 & 0.15 & 46.7 & 6433 & 0.36 & 75.0 & ns & 0.0004 & ns \\
\hline 4 & CSF & 5865 & 0.31 & 100.0 & 5867 & 0.11 & 40.0 & 5866 & 0.11 & 50.0 & $<0.0001$ & $<0.0001$ & ns \\
\hline 5 & met & - & - & 0 & 4418 & 0.29 & 33.3 & 4418 & 0.10 & 25.0 & 0.006 & 0.0219 & ns \\
\hline 6 & met & 4816 & 0.19 & 52.2 & 4819 & 0.24 & 46.7 & 4819 & 0.16 & 41.7 & ns & ns & ns \\
\hline 7 & met & 6035 & 0.02 & 17.4 & 6025 & 0.23 & 40.0 & 6024 & 0.04 & 12.5 & ns & ns & ns \\
\hline 8 & $\mathrm{gbm}$ & - & - & 0 & 1866 & 0.09 & 20.0 & 1865 & 0.23 & 29.2 & ns & 0.0094 & ns \\
\hline 9 & met & - & - & 0 & 3476 & 0.21 & 26.7 & 3475 & 0.18 & 25.0 & 0.0185 & 0.0219 & ns \\
\hline
\end{tabular}

rel int = relative intensity; $\mathrm{CSF}=$ cerebrospinal fluid; $\mathrm{gbm}=$ glioblastoma; met = metastases. $\mathrm{p}$-values obtained from a two-sided Fishers exact test of a $2 \mathrm{x} 2$ contingency table. $n s=$ not significant $(\mathrm{p}>0.05)$. 
(HPA051518, Sigma-Aldrich) the immunogen consists of the amino acids 28 to 77 of the protein, mainly corresponding to the processed truncated apolipoprotein C1 (aa29 to 83, 6432 Dalton). For anti-LuzP6 a custom-made antibody was purchased, the immunogen consisted of the 29 n-terminal amino acids (MKSVISYALYQVQTGSLPVYSSVLTKSPL) of the LuzP6 protein (Abnova, Taiwan). The paraffin slides were processed according to standard protocols and automated staining was performed in a Benchmark Ultra 2 (Ventana). After deparaffinization, the slices for ApoC1 staining were preconditioned in CC1 buffer for 36 minutes. All further steps took place using the standard reaction buffer $(\mathrm{pH} 7.4$ to 7.8$)$ at $37^{\circ} \mathrm{C}$. The antibodies were diluted 1:50 in antibody diluent (Ventana) and incubated for 60 minutes. Visualization was performed with the Ultra view universal DAB detection kit followed by a hematoxylin/bluing counterstaining.

\section{Results}

The heterogeneity of peaks was lowest in CSF from controls (94 bands from 23 samples, ca. 4/sample), average in cyst fluid from glioblastoma (148 from 24 samples, ca. 6/sample) and highest in metastases (125 peaks from 15 samples, ca. 8/sample). Altogether, 367 peaks could be distinguished and listed according to their relative intensity.

Theoretically, low mass peaks of high intensity can be generated from larger proteins bearing more than one charge $(z>1)$ or from multi-homomeric proteins that are disassembled during the ionization process. To address this issue, the $\mathrm{m} / \mathrm{z}$ ratio of the smaller bands was checked for the presence of peaks corresponding to multiples of their size (Table 2). Concerning proteins shown in table 1, this was the case for the peaks at 3513 and 5865 (both CSF), 6025 (met) and 1865 (gbm).

According to this, the protein peak 4 (m/z 5865) is likely to stem from a physiologic CSF protein (peak 16, m/z 11730), since both peaks are found in every CSF sample and in about half of all tumor samples, with significantly different frequencies between diagnosis groups (Table 2). In peaks 4 and 16, the relative intensities of the low weight peaks are about twice the intensity of the higher weight peaks. Among potential proteins fitting into this relation beta-microglobulin $(11,731 \mathrm{Da})$, a major histocompatibility complex class-I associated protein present on all nucleated cells [3]; antileukoproteinase (11,726 Da), a secreted proteinase inhibitor involved in NF-kappa-B regulation; and the $\mathrm{n}$-cym protein $(11,733 \mathrm{Da})$, which is expressed in fetal brain, cerebral neuronal cells and neuroblastoma can be found.

A similar, but less significant relation is found for the metastasis peak pair at $\mathrm{m} / \mathrm{z} 6025$ and 12,050 (Table 2). Both peaks occur predominantly in metastases, and in 4 of the metastasis samples (three of them are lung cancer metastases) both peaks are present. These findings match very well with the protein oncomodulin-1, which can bind two calcium ions, and which has been shown to be 
Table 2. MALDI-TOF MS protein peaks with related bands that are multiples of their size within the range of $+/-0.3 \%$. "Nr." gives the rank of the peak according to its relative intensity out of 367.

\begin{tabular}{|c|c|c|c|c|c|c|c|c|c|c|c|c|c|c|}
\hline \multirow{2}{*}{ Nr. } & \multirow{2}{*}{ times/size } & \multirow{2}{*}{ found in } & \multicolumn{3}{|c|}{ CSF } & \multicolumn{2}{|c|}{ metastases } & \multicolumn{4}{|c|}{ glioblastoma } & \multicolumn{3}{|c|}{$\mathrm{p}$-value } \\
\hline & & & Dalton & rel int & $\%(\mathrm{n}=23)$ & Dalton & rel int & $\%(\mathrm{n}=15)$ & Dalton & rel int & $\%(\mathrm{n}=24)$ & CSF-met & CSF-gbm & met-gbm \\
\hline 1 & 3513 & CSF & 3513 & 0.86 & 91.3 & 3511 & 0.05 & 13.3 & 3511 & 0.07 & 12.5 & $<0.0001$ & $<0.0001$ & ns \\
\hline 68 & $\times 27026$ & CSF & 7028 & 0.04 & 13.0 & - & - & 0 & 7017 & 0.004 & 8.3 & ns & ns & ns \\
\hline 127 & $\times 414,052$ & met & 14,085 & 0.01 & 13.0 & 14,090 & 0.01 & 20.0 & 14,060 & 0.01 & 16.7 & ns & ns & ns \\
\hline 4 & 5865 & CSF & 5865 & 0.31 & 100.0 & 5867 & 0.11 & 40.0 & 5866 & 0.11 & 50.0 & $<0.0001$ & $<0.0001$ & ns \\
\hline 16 & $\times 211,730$ & CSF & 11,727 & 0.16 & 100.0 & 11,732 & 0.06 & 40.0 & 11,731 & 0.05 & 62.5 & $<0.0001$ & 0.0016 & ns \\
\hline 7 & 6025 & met & 6035 & 0.02 & 17.4 & 6025 & 0.23 & 40.0 & 6024 & 0.04 & 12.5 & ns & ns & ns \\
\hline 21 & $\times 212,050$ & met & - & - & 0 & 12043 & 0.13 & 26.7 & 12036 & 0.01 & 8.3 & 0.0185 & ns & ns \\
\hline 8 & 1865 & gbm & - & - & 0 & 1866 & 0.09 & 20.0 & 1865 & 0.23 & 29.2 & ns & 0.0094 & ns \\
\hline 94 & $\times 23730$ & met & - & - & 0 & 3731 & 0.02 & 6.7 & 3741 & 0.001 & 4.2 & ns & ns & ns \\
\hline 102 & $\times 47460$ & met & - & - & 0 & 7445 & 0.02 & 13.3 & 7458 & 0.02 & 25 & ns & 0.0219 & ns \\
\hline 113 & $\times 611,190$ & met & - & - & 0 & 11,188 & 0.02 & 6.7 & - & - & 0 & ns & ns & ns \\
\hline 194 & $\times 814,920$ & met & - & - & 0 & 14,966 & 0.002 & 6.7 & 14,937 & 0.0002 & 4.2 & ns & ns & ns \\
\hline
\end{tabular}

rel int = relative intensity; CSF = cerebrospinal fluid; $\mathrm{gbm}=$ glioblastoma; met = metastases. $\mathrm{p}$-values obtained from a two-sided Fishers exact test of a $2 \times 2$ contingency table. $n s=$ not significant $(\mathrm{p}>0.05)$.

associated with a metastatic phenotype of cancer cells [4] [5]. These peaks had not been detected by the former SELDI-TOF analysis (Table 4).

For the glioblastoma peak m/z 1865 and its quadruple 7460 a significant difference to occurrence in CSF is found (Table 2). However, neither for $\mathrm{m} / \mathrm{z} 1865$ nor for its double 3730 Expasy tag-ident results are obtained. For m/z 7460 about a dozen protein candidates are found, mostly isoforms of larger proteins, such as chemotactic factors, but also the insulin-like growth factor $2(\mathrm{~m} / \mathrm{z} 7476)$ [6].

Only the octuple size of $\mathrm{m} / \mathrm{z} 1865(14,946 \mathrm{Da})$ had been detected using SELDI-TOF MS, with a significant difference between glioblastoma cyst fluid and CSF (Table 3). Potential proteins are interleukin-4 (14,963 Da), Profilin 1 and $2(14,923$ and 14,915 Da), as well as cystatin-9 (14,943 Da). Of special interest might be Maturin (14,925 $\mathrm{Da}$, C7orf41), a protein involved in early neuronal development [7].

The peak at $\mathrm{m} / \mathrm{z} 3513$ remains very difficult to interpret. Though it has the highest relative intensity of all peaks and occurs significantly more frequent in CSF samples than in glioblastoma or metastasis, no corresponding tagident results can be obtained. Due to its small size, the multiple of sizes open access to a huge number of potential proteins, but the peaks at m/z 7026 and 14,052 neither are very prominent, nor do they show a significantly different distribution between diagnosis groups (Table 2). Potential proteins are the "Negative regulator of P-body association" (7025 Da) and RPABC4 (7004 Da), a subunit of DNA-directed RNA polymerases, as well as the chain B of chymotrypsinogen $B$ (14,049 Da). 
Table 3. MS result synopsis for the comparison between glioblastoma cyst fluid and CSF. 21 protein peaks described by SELDI-TOF analysis were found in the present MALDI-TOF analysis, 4 of them (m/z 6433, 6632, 13879 and 16630) again with significant difference between diagnosis groups.

\begin{tabular}{|c|c|c|c|c|c|c|c|c|}
\hline \multicolumn{5}{|c|}{ SELDI-TOF MS [2] } & \multirow[b]{3}{*}{$P$-value } & \multicolumn{3}{|c|}{ MALDI-TOF MS (present study) } \\
\hline \multicolumn{3}{|c|}{ Glioblastoma versus CSF } & \multicolumn{2}{|c|}{ CSF versus glioblastoma } & & \multicolumn{2}{|c|}{ Glioblastoma versus CSF } & \multirow[b]{2}{*}{$P$-value } \\
\hline signal found in & MW (Da) & $P$-value & signal found in & MW (Da) & & signal mainly found in & MW (Da) & \\
\hline & & & & & & & in $\mathrm{gbm}$ & CSF vs gbm \\
\hline gbm cyst fluid & 6424 & $4.42 \times 10^{-7}$ & & & & gbm cyst fluid & 6433 & $4.00 \times 10^{-4}$ \\
\hline \multirow[t]{5}{*}{ gbm cyst fluid } & 6633 & $1.53 \times 10^{-5}$ & & & & gbm cyst fluid & 6632 & $1.89 \times 10^{-2}$ \\
\hline & & & CSF & 6799 & $1.08 \times 10^{-3}$ & metastasis & 6802 & ns \\
\hline & & & CSF & 6888 & $1.47 \times 10^{-4}$ & CSF & 6893 & ns \\
\hline & & & CSF & 6936 & $3.82 \times 10^{-6}$ & CSF & 6932 & ns \\
\hline & & & CSF & 7647 & $5.24 \times 10^{-6}$ & CSF & 7653 & ns \\
\hline gbm cyst fluid & 7921 & $3.46 \times 10.2$ & & & & gbm cyst fluid & 7933 & ns \\
\hline gbm cyst fluid & 8687 & $3.82 \times 10^{-6}$ & & & & metastasis & 8688 & ns \\
\hline gbm cyst fluid & 9362 & $3.26 \times 10^{-6}$ & & & & gbm cyst fluid & 9358 & ns \\
\hline gbm cyst fluid & 9418 & $3.26 \times 10^{-6}$ & & & & metastasis & not found & ns \\
\hline gbm cyst fluid & 9948 & $4.42 \times 10^{-7}$ & & & & CSF & 9964 & ns \\
\hline gbm cyst fluid & 10,829 & $5.56 \times 10^{-3}$ & & & & gbm cyst fluid & 10,829 & ns \\
\hline \multirow[t]{4}{*}{ gbm cyst fluid } & 12,559 & $2.77 \times 10^{-6}$ & & & & CSF & not found & ns \\
\hline & & & CSF & 13,687 & $3.69 \times 10^{-5}$ & metastasis & not found & ns \\
\hline & & & CSF & 13,737 & $2.18 \times 10^{-4}$ & CSF & not found & ns \\
\hline & & & CSF & 13,879 & $9.74 \times 10^{-6}$ & CSF & not found & $9.40 \times 10^{-3}$ \\
\hline gbm cyst fluid & 14,946 & $4.08 \times 10^{-3}$ & & & & metastasis & 14,937 & ns \\
\hline gbm cyst fluid & 15,118 & $1.00 \times 10^{-2}$ & & & & gbm cyst fluid & 15,127 & ns \\
\hline gbm cyst fluid & 15,842 & $9.12 \times 10^{-3}$ & & & & metastasis & 15,866 & ns \\
\hline gbm cyst fluid & 16,656 & $1.33 \times 10^{-2}$ & & & & CSF & 16,630 & $1.71 \times 10^{-2}$ \\
\hline gbm cyst fluid & 28,056 & $1.61 \times 10^{-5}$ & & & & gbm cyst fluid & 28,025 & ns \\
\hline
\end{tabular}

CSF: cerebrospinal fluid; gbm: glioblastoma; MW (Da) : molecular weight in Dalton. P-value: level of significance in exponential form. Ns: not significant.

The size of peaks detected by MALDI-TOF ranged from m/z 1184 to 33,276. Below $\mathrm{m} / \mathrm{z} 1400$ and above 23,000 the peaks occurred merely in one or two samples of a diagnosis group and with only very low relative intensities $(<0.03)$. This detection range is more restricted in comparison to the SELDI-TOF analysis ( $\mathrm{m} / \mathrm{z} 3892$ to 146,376) but allows for description of smaller proteins.

In the SELDI-TOF MS analysis, 51 peaks had occurred with significant difference between glioblastoma and CSF [2]. 21 of these protein peaks were also identified in the present analysis using MALDI-TOF (Table 3). 12 peaks (57\%) were retrieved in the same kind of fluid (glioblastoma cyst or CSF), comprising 3 in which the significant difference between glioblastoma cyst fluid and CSF could be confirmed. 
When comparing metastasis cyst fluid with CSF, 49 peaks had been found by SELDI-TOF MS analysis and 20 of them were confirmed in the present study by MALDI-TOF MS (Table 4). Again, more than half of the peaks were retrieved in the same diagnosis group (11 peaks, 55\%). Two peaks were confirmed with significantly different frequency between cyst fluid and CSF.

Among the SELDI-TOF peaks that were confirmed by MALDI-TOF (Table 3 and Table 4), 14 are present in both comparisons (glioblastoma versus CSF and metastasis versus CSF). 10 (71\%) remain in the same diagnosis group (tumor or CSF).

In CSF, three protein peaks were found with a significantly different number of positive samples between diagnosis groups, two when comparing CSF to glioblastoma cyst fluid (m/z 13,879 and 16,630), and one in comparison to metastases $(\mathrm{m} / \mathrm{z} 11,732)$. The latter is most likely associated to beta-microglobulin as discussed above (peak 16, Table 2).

Table 4. MS result synopsis for the comparison between metastasis cyst fluid and CSF. 20 protein peaks described by SELDI-TOF analysis were found in the present MALDI-TOF analysis, 2 of them (m/z 9419 and 11,732) again with significant difference between the diagnosis groups.

\begin{tabular}{|c|c|c|c|c|c|c|c|c|}
\hline \multicolumn{5}{|c|}{ SELDI-TOF MS [2] } & \multirow[b]{3}{*}{$P$-value } & \multicolumn{3}{|c|}{ MALDI-TOF MS (present study) } \\
\hline \multicolumn{3}{|c|}{ Metastasis versus CSF } & \multicolumn{2}{|c|}{ CSF versus metastasis } & & \multirow{2}{*}{$\begin{array}{l}\text { Metastasis versus CSF } \\
\text { signal mainly found in }\end{array}$} & \multirow{2}{*}{ MW (Da) } & \multirow[b]{2}{*}{$P$-value } \\
\hline signal found in & MW (Da) & $P$-value & signal found in & MW (Da) & & & & \\
\hline & & & & & & & in metastasis & CSF vs met \\
\hline & & & CSF & 3892 & $1.08 \times 10^{-4}$ & CSF & not found & ns \\
\hline metastasis & 4153 & $3.03 \times 10^{-3}$ & & & & CSF & not found & ns \\
\hline metastasis & 5099 & $9.52 \times 10^{-4}$ & & & & metastasis & 5102 & ns \\
\hline \multirow[t]{5}{*}{ metastasis } & 6436 & $4.90 \times 10^{-3}$ & & & & gbm cyst fluid & 6435 & ns \\
\hline & & & CSF & 6799 & $3.10 \times 10^{-4}$ & metastasis & 6804 & ns \\
\hline & & & CSF & 6888 & $2.31 \times 10^{-4}$ & CSF & 6888 & $\mathrm{~ns}$ \\
\hline & & & CSF & 6938 & $9.28 \times 10^{-5}$ & CSF & 6940 & ns \\
\hline & & & CSF & 7025 & $5.35 \times 10^{-6}$ & CSF & not found & ns \\
\hline metastasis & 7943 & $3.95 \times 10^{-2}$ & & & & gbm cyst fluid & 7936 & ns \\
\hline metastasis & 9423 & $9.11 \times 10^{-6}$ & & & & metastasis & 9419 & $1.85 \times 10^{-2}$ \\
\hline \multirow[t]{2}{*}{ metastasis } & 11,278 & $4.14 \times 10^{-4}$ & & & & metastasis & 11,307 & ns \\
\hline & & & CSF & 11704 & $2.58 \times 10^{-6}$ & CSF & 11,732 & $<1 \times 10^{-5}$ \\
\hline \multirow[t]{4}{*}{ metastasis } & 12,535 & $2.54 \times 10^{-5}$ & & & & CSF & not found & ns \\
\hline & & & CSF & 13688 & $3.10 \times 10^{-4}$ & metastasis & 13,651 & $\mathrm{~ns}$ \\
\hline & & & CSF & 13741 & $2.31 \times 10^{-4}$ & CSF & not found & ns \\
\hline & & & CSF & 13871 & $2.08 \times 10^{-3}$ & CSF & 13,879 & $\mathrm{~ns}$ \\
\hline metastasis & 15,116 & $4.90 \times 10^{-3}$ & & & & gbm cyst fluid & 15,139 & ns \\
\hline metastasis & 15,866 & $9.52 \times 10^{-4}$ & & & & metastasis & 15,891 & $\mathrm{~ns}$ \\
\hline metastasis & 16,621 & $1.71 \times 10^{-4}$ & & & & CSF & 16,645 & ns \\
\hline metastasis & 28,081 & $4.75 \times 10^{-2}$ & & & & gbm cyst fluid & not found & ns \\
\hline
\end{tabular}

CSF: cerebrospinal fluid; gbm: glioblastoma; met: metastasis. MW (Da): molecular weight in Dalton. P-value: level of significance in exponential form. Ns: Not significant. 
The peak at m/z 13879 has not been detected in glioblastoma cyst fluid, it is present in CSF and metastases with about similar percentage of positive samples (Table 3 and Table 4). This constellation allows the hypothetical suggestion of a protein that is lost during pathogenetic development of glioblastoma.

The protein peak at $\mathrm{m} / \mathrm{z} 16,630$ had first been detected in glioblastoma cyst fluid by SELDI-TOF MS (m/z 16,656), but was predominantly found in CSF in the MALDI-TOF MS examination (Table 3 ). The same situation was encountered in metastases versus CSF. SELDI-TOF MS showed a peak at $\mathrm{m} / \mathrm{z} 16,621$ in metastasis cyst fluid, but MALDI-TOF MS detected this peak in CSF (m/z 16645, Table 4). Among the many potential proteins displayed in tagident when searching with this rather high $\mathrm{m} / \mathrm{z}$ ratio, is the neuroendocrine protein $7 \mathrm{~B} 2$ (16,640 Da, “secretogranin 5"), a molecular chaperone involved in transport and secretion of neuropeptides, that has been shown to be down-regulated by DNA methylation in astrocytic gliomas [8]. Other corresponding proteins are probetacellulin $(16,629 \mathrm{Da})$, a growth factor that binds to the EGF-receptor, and Eukaryotic translation initiation factor $5 \mathrm{~A}-2(16,662 \mathrm{Da})$, a protein involved in mRNA turnover and cell cycle progression.

In the metastasis cyst samples a protein peak at m/z 9419 was found with both techniques (MALDI and SELDI), with a significantly higher percentage of positive samples in metastasis cyst fluids than in CSF (Table 4, MALDI: $p=1.85 \times$ $10^{-2}$ ). The peak had been present in the SELDI-analysis of glioblastoma cyst fluid, but was not confirmed by MALDI-TOF MS (Table 3). A protein supporting the metastasizing phenotype might be underlying, but among potential protein candidates are also C-X-C motif chemokine 14, a secreted chemoattractant for neutrophils expressed in brain, parathyroid hormone and $40 \mathrm{~S}$ ribosomal protein S27a, that can be linked to ubiquitin.

In glioblastoma cyst fluid, MALDI-TOF MS confirmed at nearly the same size and with significant different occurrence to CSF and to metastases the peak at $\mathrm{m} / \mathrm{z} 6632$ (Table 3). This size may correspond to the SH2 domain-containing protein $1 \mathrm{~A}(6631 \mathrm{Da})$, a factor involved in lymphocytic immune response, the C-X-C motif chemokine $17(6627 \mathrm{Da})$, playing a role in angiogenesis and cell differentiation, or the protein ApoC1. ApoC1 is coded on chromosome $19 q 13.32$, it is involved in lipoprotein metabolism and because of alternative splicing many transcript variants exist. The gene is activated when monocytes differentiate into macrophages and ApoC1 expression has been shown in cerebral tissue [9].

ApoC1 is also found, when searching the Expasy tagident database for the most prominent glioblastoma cyst peak at $\mathrm{m} / \mathrm{z}$ 6433. SELDI-TOF MS had detected the peak in glioblastomas $(\mathrm{m} / \mathrm{z}$ 6424) and in metastases $(\mathrm{m} / \mathrm{z}$ 6436), MALDI-TOF MS analysis now narrowed the range to lie between $\mathrm{m} / \mathrm{z} 6433$ and 6435 (Table 3 and Table 4). Because of the many splice variants, ApoC1 is listed at $6631 \mathrm{Da}$ and a truncated ApoC1 form exists at a molecular weight of $6432 \mathrm{Da}$. Only one other protein is displayed by tagident at this size, the Leucine zipper 
protein $6(6437 \mathrm{Da})$. This protein is possibly a self-tumor antigen, associated to myeloproliferative disease. The gene is located on chromosome $7 \mathrm{q} 33$ with an atypical AUU translation start codon in the 3'-untranslated region of the myotrophin gene [10].

To further assess cellular expression of the two proteins in tumor tissue, immunohistochemistry of histologic slides of cystic glioblastoma was performed. In addition to the physiologically expressed ApoC1, leucine zipper protein 6 could also be detected by antibody staining, thus supporting the results of the spectrometric analyses (Figure 2).

\section{Discussion}

MALDI-TOF and SELDI-TOF MS are discovery-oriented approaches and deliver a multitude of results, that have to be sorted and analyzed for further assessment. In the present publication, we were able to refine the former SELDI-TOF MS results by employing MALDI-TOF MS assessment of brain tumor cyst fluid in a new set of fluid specimens. The range of protein sizes discovered by MALDI-TOF MS is smaller and more narrow, mainly between 2 and $20 \mathrm{kDa}$. Given the $+/-0.3 \%$ accuracy range of both methods, 41 protein peaks have been re-identified when comparing CSF with cyst fluid from glioblastoma or metastases, and more than half of the peaks occurred in the same diagnosis group, namely tumor or CSF.

In 6 protein peaks, significantly different numbers of positive samples between the diagnosis groups were confirmed. Only one of these peaks ( $\mathrm{m} / \mathrm{z} 16,621$ to 16,656$)$ underwent a diagnosis shift. Whereas in the SELDI-TOF MS analysis it was predominantly present in glioblastoma cyst fluid or metastasis cyst fluid, MALDI-TOF MS showed its occurrence in CSF.

This diagnosis shift displays two limitations of the approach. On one hand, if an important part of the cyst fluid consists of CSF, as for example in recurrent tumors with large residual resection cavities, the protein content will be attributed to the (recurrent) tumor, following the informations received from the neurosurgeon after fluid aspiration, though it may mainly contain CSF.



Figure 2. Immunohistochemical staining of corresponding slides of a cystic glioblastoma. (A) ApoC1 staining with hematoxylin/bluing counterstain: few positive tumor cells and endothelial expression; (B) LuzP6 (n-terminal) staining with hematoxylin/bluing counterstain: a high number of positive tumor cells showing nuclear expression (original magnification $200 \times$ ). 
On the other hand, high molecular weights are difficult to analyze, since our analysis algorithm allows for a deviation of $0.3 \%$ according to the methods accuracy, which at this size means up to 50 Dalton. The potential proteins underlying this peak can thus differ by more than 10 amino acids, leading to a blurred way of identification. Moreover, at this size the Expasy tagident tool displays regularly more than 50 possibly "discovered" proteins, among them many isoforms, truncated, or even only inferred proteins. Lastly, the status of cysteine residue oxidation remains unknown, as well as the intratumoral pH-value. From this point of view, the restriction of the MALDI-TOF MS approach to smaller protein sizes may enable an easier identification.

Concerning the other 5 protein peaks with confirmed significant difference between tumor samples and CSF, two might be physiological CSF proteins $(\mathrm{m} / \mathrm{z}$ 11,727 and 13,879). One is assigned to metastasis tissue (9419 Da) and had also been found in glioblastoma cyst fluid by SELDI-TOF MS analysis. Since brain metastases arise from different primary tumors, it can be speculated that the underlying protein constitutes a part of the cerebral immune response against the growing cells, such as the secreted chemotactic factor C-X-C motif chemokine 14.

The two remaining significant protein peaks (m/z 6433 and 6632) are found in glioblastoma cyst fluid compared to CSF. In accordance to the SELDI-TOF MS results, the peak at $\mathrm{m} / \mathrm{z} 6433$ was again the most predominant, and the more precise mass determination by MALDI-TOF MS reduced the list of potential candidates down to apolipoprotein $\mathrm{C} 1$ and leucine zipper protein 6. Searching the tagident tool with $\mathrm{m} / \mathrm{z} 6632$ resulted in 10 potential proteins, among them a further splice variant of ApoC1. Immunohistochemical staining then confirmed the presence of both proteins in tissue of cystic glioblastoma (Figure 2), thus underlining the feasibility of the analysis algorithm to detect proteins that are connected to glioblastoma metabolic pathways.

Whereas ApoC1 is known to be expressed in cerebral tissue [9], the LuzP6 results can only be interpreted with a hypothetical explanation. The protein myotrophin interferes with NFkappaB regulation, that may play a role in glioblastoma growth [11]. LuzP6 is translated from the myotrophin gene, which is known to be stimulated by insulin like growth factors [12] [13]. The protein size of insulin-like growth factor $2(7458 \mathrm{Da})$ has been detected in the present MALDI-TOF MS analysis, significantly more frequent in glioblastoma cyst fluid than in CSF (Table 2). LuzP6 may then be expressed as a result of this stimulation, or in form of a fusion protein with myotrophin [14].

Our ongoing research deals with immunohistochemical analysis of a larger number of glioblastoma specimens including non-cystic tumors, to identify the percentage of LuzP6 expression in comparison to ApoC1, and in relation to further candidate proteins. Immunostaining of myotrophin, together with mRNA analysis of protein expression will be needed, to elucidate potential growth stimulating metabolic pathways. 
The present results have refined and focused the former SELDI-TOF MS analysis using MALDI-TOF MS and are confirmed by immunohistochemistry. They support the proteomic approach as a way to generate hypotheses on growth stimulating pathways in brain tumors, aiming for the discovery of tumor-associated biomarkers [15].

\section{Conclusion}

Mass spectrometric analysis by laser-desorption ionization is a feasible tool to assess brain tumor cyst protein content in comparison to cerebrospinal fluid. A thorough work-up, including internet-based data research, immunohistochemistry and mRNA-analysis is needed to describe the obtained results in detail and to develop hypotheses concerning biomarkers. In the present study, immunohistochemistry confirmed the presence of apolipoprotein $\mathrm{C} 1$ and leucine zipper protein 6 in glioblastoma tissue, as supposed from MALDI-TOF MS assessment.

\section{References}

[1] Stockhammer, G., Obwegeser, A., Kostron, H., et al. (2000) Vascular Endothelial Growth Factor (VEGF) Is Elevated in Brain Tumor Cysts and Correlates with Tumor Progression. Acta Neuropathologica, 100, 101-105.

https://doi.org/10.1007/s004010051199

[2] Hoelscher, M., Richter, N., Melle, C., et al. (2013) SELDI-TOF Analysis of Glioblastoma Cyst Fluid Is an Approach for Assessing Cellular Protein Expression. Neurological Research, 35, 993-1001. https://doi.org/10.1179/016164113X13756993777580

[3] Svatoňová, J., Bořecká, K., Adam, P., et al. (2014) Beta2-Microglobulin as a Diagnostic Marker in Cerebrospinal Fluid: A Follow-Up Study. Disease Markers, 495402. https://doi.org/10.1155/2014/495402

[4] Brewer, L.M., Durkin, J.P. and MacManus, J.P. (1984) Immunocytochemical Detection of Oncomodulin in Tumor Tissue. Journal of Histochemistry \& Cytochemistry, 32, 1009-1016. https://doi.org/10.1177/32.10.6384359

[5] Nestl, A., Von Stein, O.D., Zatloukal, K., et al. (2001) Gene Expression Patterns Associated with the Metastatic Phenotype in Rodent and Human Tumors. Cancer Research, 61, 1569-1577.

[6] Weroha, S.J. and Haluska, P. (2012) The Insulin-Like Growth Factor System in Cancer. Endocrinology and Metabolism Clinics of North America, 41, 335-350. https://doi.org/10.1016/j.ecl.2012.04.014

[7] Martinez-De Luna, R.I., Ku, R.Y., Lyou, Y., et al. (2013) Maturin Is a Novel Protein Required for Differentiation during Primary Neurogenesis. Developmental Biology, 384, 26-40. https://doi.org/10.1016/j.ydbio.2013.09.028

[8] Waha, A., Felsberg, J., Hartmann, W., et al. (2012) Frequent Epigenetic Inactivation of the Chaperone SGNE1/7B2 in Human Gliomas. International Journal of Cancer, 131, 612-622. https://doi.org/10.1002/ijc.26416

[9] Cudaback, E., Li, X., Yang, Y., et al. (2012) Apolipoprotein C-I Is an APOE Genotype-Dependent Suppressor of Glial Activation. Journal of Neuroinflammation, 9 , 192. https://doi.org/10.1186/1742-2094-9-192

[10] Xiong, Z., Liu, E., Yan, Y., et al. (2006) An Unconventional Antigen Translated by a Novel Internal Ribosome Entry Site Elicits Antitumor Humoral Immune Reactions. 
The Journal of Immunology, 177, 4907-4916. https://doi.org/10.4049/jimmunol.177.7.4907

[11] Knuefermann, P., Chen, P., Misra, A., et al. (2002) Myotrophin/V-1, a Protein Up-Regulated in the Failing Human Heart and in Postnatal Cerebellum, Converts NFkappa B p50-p65 Heterodimers to p50-p50 and p65-p65 Homodimers. The Journal of Biological Chemistry, 277, 23888-23897. https://doi.org/10.1074/jbc.M202937200

[12] Peters, G., Gongoll, S., Langner, C., et al. (2003) IGF-1R, IGF-1 and IGF-2 Expression as Potential Prognostic and Predictive Markers in Colorectal-Cancer. Virchows Archiv, 443, 139-145. https://doi.org/10.1007/s00428-003-0856-5

[13] Suvasini, R., Shruti, B., Thota, B., et al. (2011) Insulin Growth Factor-2 Binding Protein 3 (IGF2BP3) Is a Glioblastoma-Specific Marker that Activates Phosphatidylinositol 3-Kinase/Mitogen-Activated Protein Kinase (PI3K/MAPK) Pathways by Modulating IGF-2. The Journal of Biological Chemistry, 286, 25882-25890. https://doi.org/10.1074/jbc.M110.178012

[14] Zumkeller, W., Sääf, M. and Rähn, T. (1993) Insulin-Like Growth Factor (IGF)-I, -II and IGF-Binding Proteins in the Cyst Fluid of a Patient with Astrocytoma. Child s Nervous System, 9, 100-103. https://doi.org/10.1007/BF00305317

[15] Kim, J.H., Lee, S.K., Yoo, Y.C., et al. (2012) Proteome Analysis of Human Cerebrospinal Fluid as a Diagnostic Biomarker in Patients with Meningioma. Medical Science Monitor, 18, BR 450-460.

\section{Abbreviations}

ACN: acetonitrile

ApoC1: apolipoprotein C1

CNS: central nervous system

CSF : cerebrospinal fluid

GBM: glioblastoma

HCCA: alpha-cyano-4-hydroxycinnamic acid

LuzP6: leucine zipper protein 6

$\mathrm{m}$ : molecular mass [Dalton]

MALDI: matrix assisted laser desorption ionization

MET: metastasis

MS: mass spectrometry

SELDI: surface enhanced laser desorption ionisation

TFA: trifluoroacetate

TOF: time of flight

z: electrical charge of a molecule 